\title{
COHERENT POPULATION TRAPPING IN OPEN AND CLOSED SYSTEMS
}

\author{
L. WINDHOLZ* \\ Institut für Experimentalphysik, Technische Universität Graz \\ Petersgasse 16, 8010 Graz, Austria \\ (Received July 7, 1999)

\begin{abstract}
In the present paper, an overview on work concerning coherent population trapping within the hyperfine manifold of the sodium $D_{1}$-line is given. Besides manifestation of coherent population trapping for different $\Lambda$-schemes, also phase dependent processes in closed contour systems (four-level double $\Lambda$-schemes) are discussed, including the case of phase dependent electromagnetically induced transparency.
\end{abstract}

PACS numbers: $32.80 .-\mathrm{t}, 42.50 .-\mathrm{p}$

\section{Introduction}

In 1976, Alzetta and co-workers (Dipartimento di Fisica, Universita di Pisa) $[1,2]$ discovered the appearance of non-excited regions within the fluorescence path while exciting sodium vapour in a fluorescence cell placed in an inhomogeneous magnetic field. The laser light used had several frequency components, emitted by a dye laser which was running simultaneously with different longitudinal modes. Six free spectral ranges of the laser resonator gives a frequency distance of approximately the hyperfine splitting of the sodium ground state $\left(3^{2} S_{1 / 2}, F=2-F=1\right)$ and fits exactly to the splitting of two Zeeman components of the hyperfine manifold in a certain spatial region of the cell (see Fig. 1). In this region the resonance fluorescence vanishes or is at least much weaker, therefore this effect was first called a "black line". Soon later, Arimondo and Orriols [3] explained this effect as an optical pumping of the atoms into a non-absorbing superposition of the ground states, which later gave rise to the name "coherent population trapping" (CPT). Independently, Gray et al. [4] observed a similar effect when exciting a weakly collimated sodium atomic beam with two independent laser frequencies from both hyperfine ground levels. Later on also the name "dark resonance" was used.

Let us consider a three-level $\Lambda$-atom (Fig. 2a), consisting of two ground and one excited levels. From the upper level, both transitions to the ground levels are dipole-allowed. Excitation of one transition leads to the well-known effect of

*e-mail: windholz@iep.tu-graz.ac.at 


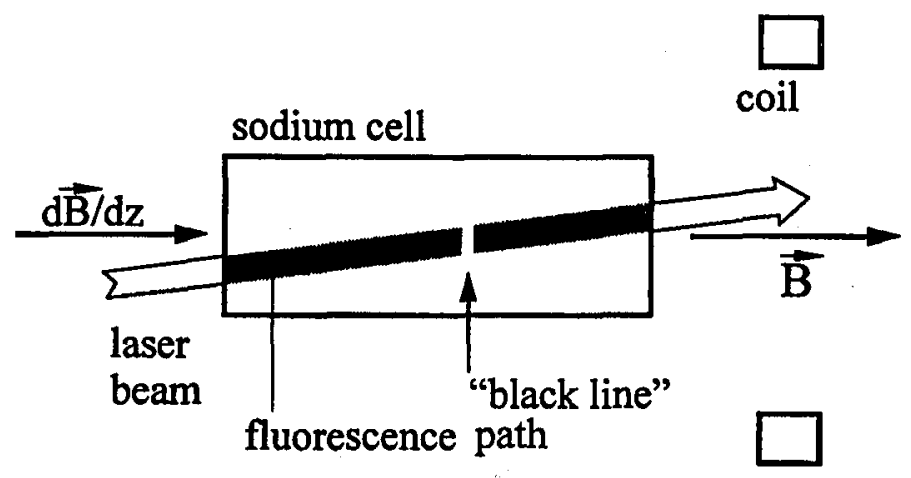

Fig. 1. Observation of a "black line" in the fluorescence path of multifrequency laser light in a sodium vapour cell. Sketch of the experiment performed by Alzetta et al. [1].

optical pumping to the other ground level, where the probability is given by the branching ratio. After some fluorescence cycles, the atoms are pumped to the second, non-excited ground level (we therefore call it a "dark state"). This optical pumping influences the number density of atoms in the excited state and thus the observed fluorescence intensity. An increase in the intensity is possible, if a second light wave is resonant with the second transition, since no atoms can now fall into a dark state. The better both exciting fields are in resonance with the transitions, the larger the population of the excited state - but surprisingly the fluorescence vanishes (the population of the upper state reaches zero), if the frequency difference between the exciting fields matches exactly the ground state frequency splitting $\nu_{12}=\left(E_{2}-E_{1}\right) / h$. In this case a Raman resonance condition

$$
\delta_{\mathrm{R}}=\left(\nu_{1}-\nu_{2}\right)-\left(\nu_{31}-\nu_{32}\right)=0
$$

(often called also a two-photon resonance condition) is fulfilled, where $\nu_{31}=$ $\left(E_{3}-E_{1}\right) / h, \nu_{32}=\left(E_{3}-E_{2}\right) / h$ are the transition frequencies, $\nu_{1}, \nu_{2}$ are the light frequencies. $\gamma_{3, i}$ in Fig. 2 indicates the spontaneous transition rates.

A theoretical treatment of such a three-level $\Lambda$-atom in a two-frequency electromagnetic field is given e.g. in the review article of Arimondo [5], using the
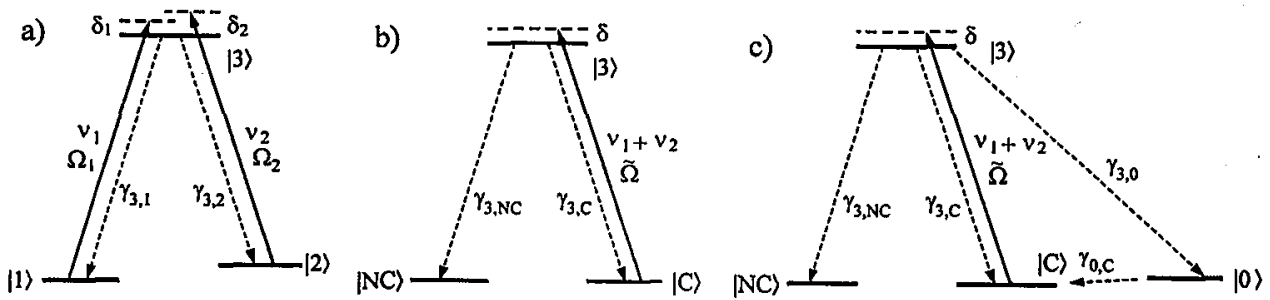

Fig. 2. Three-level $\Lambda$-scheme: (a) using eigenstates of the atomic Hamiltonian, (b) using coherent superposition states, (c) three-level $\Lambda$-scheme with losses to an additional state $|0\rangle . \nu-$ light frequencies, $\Omega-$ Rabi frequencies, $\delta$ - detunings, $\gamma-$ spontaneous decay rates. 
density matrix formalism. The energy due to atom-field interaction is given by the Hamiltonian

$$
V_{A L}=\hbar \frac{\Omega_{1}}{2 \mathrm{e}^{\mathrm{i}\left(\omega_{1} t+\phi_{1}\right)}}|3\rangle\left\langle 1\left|+\hbar \frac{\Omega_{2}}{2 \mathrm{e}^{-\mathrm{i}\left(\omega_{2} t+\phi_{2}\right)}}\right| 3\right\rangle\langle 2|+\text { h.c. }
$$

$\Omega_{i}=d_{k i} \mathcal{E}_{i} / \hbar$ are the Rabi frequencies measuring the strengths of the exciting fields, where $d_{k i}$ are the dipole moments, $\mathcal{E}_{i}$ are the amplitudes of the light fields.

The time evolution of the density matrix $\rho(t)$ then is given by

$$
\mathrm{i} \frac{\mathrm{d} \rho(t)}{\mathrm{d} t}=\frac{1}{\hbar}\left[H_{0}+V_{A L}, \rho(t)\right]+R \rho(t),
$$

where $H_{0}$ is the Hamiltonian of the unperturbed atom and relaxations between the different levels are taken into account by the relaxation superoperator $R$. The set of differential equations is usually solved numerically. In Fig. 3a the result for the steady state population of the excited state $|3\rangle, \rho_{33}$, is given in dependence on the Raman detuning $\delta_{R}$, showing indeed population $\rho_{33}=0$ for $\delta_{R}=0$. But this numerical solution does hardly allow a physical understanding of the effect.

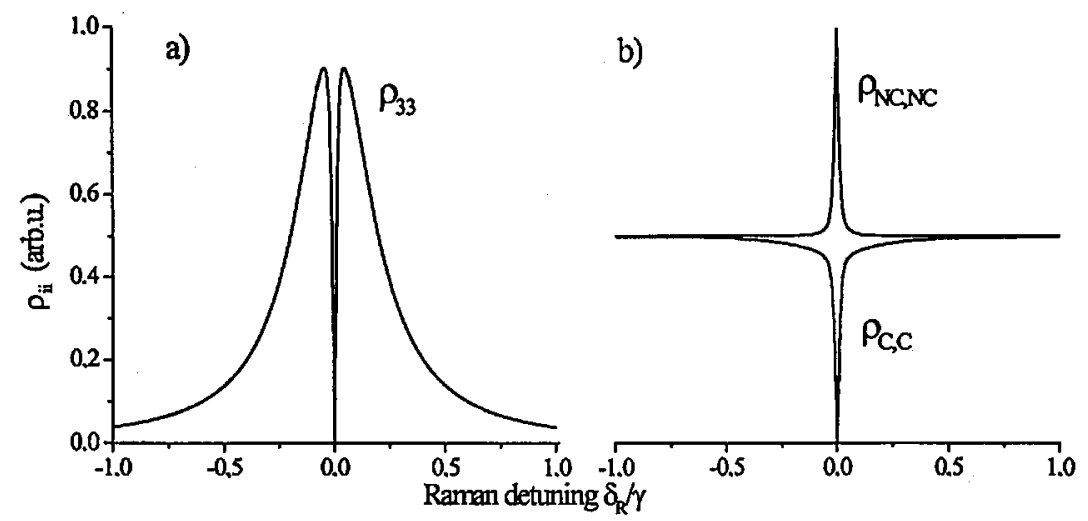

Fig. 3. Numerical solution of the equations for the density matrix elements (presentation is similar as in Ref. [5]): (a) the population $\rho_{33}$ of the upper state is zero for a Raman detuning $\delta_{\mathrm{R}}=0$; (b) additionally, the populations of the "coupled" state $|C\rangle$ and the "non-coupled" state $|N C\rangle$ are shown.

The state vector of the atom can be treated not only in the usual basis $|1\rangle,|2\rangle,|3\rangle$, but also in another basis, constructed as coherent orthogonal superpositions

$$
\begin{aligned}
& |N C\rangle=\left(\Omega_{2} / \tilde{\Omega}\right)|1\rangle-\left(\Omega_{1} / \tilde{\Omega}\right)|2\rangle, \\
& |C\rangle=\left(\Omega_{1}^{*} / \tilde{\Omega}\right)|1\rangle+\left(\Omega_{2}^{*} / \tilde{\Omega}\right)|2\rangle, \quad|3\rangle=|3\rangle,
\end{aligned}
$$

where $\tilde{\Omega}=\sqrt{\left|\Omega_{1}\right|^{2}+\left|\Omega_{2}\right|^{2}}$ (Fig. 2b). When calculating the transition matrix elements, for $\delta_{\mathrm{R}}=0$ one gets $\left\langle 3\left|V_{A L}\right| N C\right\rangle \equiv 0$ and the time development of the population $\rho_{N C, N C}$, given by $\frac{\mathrm{d}}{\mathrm{d} t}\left(\rho_{N C, N C}\right)$, is identical to zero. Atoms already being in state $|N C\rangle$ or being pumped into this state by spontaneous decay from $|3\rangle$, will 
stay there since the atoms undergo no interaction with the fields. $|N C\rangle$ therefore is a dark state for our two-frequency field, and the trapping of population in this "non-coupled" state is called coherent population trapping. The other basis state, $|C\rangle$, is coupled strongly to the exciting fields, so all the population is pumped to the coherent dark state $|N C\rangle$ after some fluorescence cycles. The populations $\rho_{N C, N C}$ and $\rho_{C, C}$ are shown in Fig. $3 \mathrm{~b}$. In this picture the effect can be seen as optical pumping to a non-absorbing coherent dark state. Another interpretation is possible when treating the dipole transition moments. In case of $\delta_{R}=0$ the phase of the transition moments is just opposite, and the common action of both exciting fields interferes to zero. A common detuning of both exciting waves is influencing the pumping rate into the coherent dark state, but not the effect itself (as long as $\delta_{\mathrm{R}}=0$ ). Therefore the effect can be seen even in fluorescence cells, where the velocity of moving atoms creates Doppler shifts which are practically the same for both transitions if they have nearly the same transition frequency. Nevertheless, most of the experiments presented here have been performed using a sodium atomic beam.

\section{Manifestation of coherent population trapping within the hyperfine manifold of the sodium $D_{1}$-line}

Even first observed by Alzetta when investigating sodium resonance lines, the situation is not so simple as may be assumed when having in mind only the well-known hyperfine structure of the sodium $D$-lines. One easily can see that a $\Lambda$-system is formed, e.g., by the hyperfine states $3^{2} S_{1 / 2}, F_{\mathrm{g}}=1, F_{\mathrm{g}}=2$, and $3^{2} P_{1 / 2}, F_{\mathrm{e}}=2$ (two components of the hyperfine spectrum of the $D_{1}$-line). But in fact one has to consider also the manifold of magnetic quantum numbers, and here no simple three-level $\Lambda$-schemes can be found. Since all experiments described forthcoming have been performed on the sodium $D_{1}$-line, we present in Fig. 4 the hyperfine manifold with quantum number designations, but will omit the quantum numbers in the following figures.

As the two ground states, one may select also two $m_{F}$ states belonging to the same $F$ value, and create the $\Lambda$-systems with only one excitation frequency.

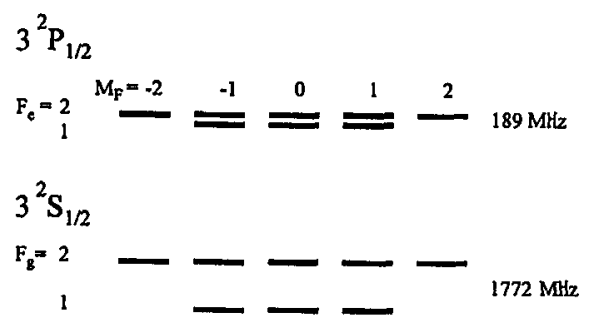

Fig. 4

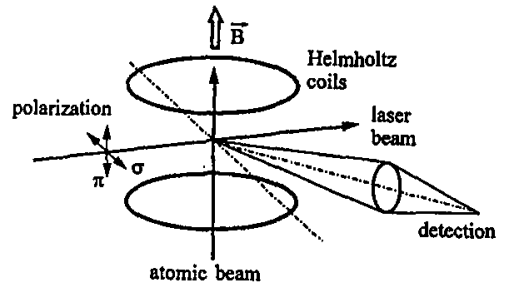

Fig. 5

Fig. 4. Magnetic substates of the sodium $D_{1}$-line.

Fig. 5. Geometrical arrangement of atomic beam, magnetic field $\boldsymbol{B}$, excitation and observation direction. 
As long as there is a (small) magnetic field present, the $m_{F}$ states are not completely degenerated, and the definition of $\sigma^{+}$and $\sigma^{-}$light makes sense. The two circular polarizations are present if linearly polarized light is used, shined to the atomic beam perpendicular to the external magnetic field, the polarization plane chosen also perpendicular to the magnetic field. The Raman resonance condition may be fulfilled when scanning the external magnetic field from negative to positive values. The geometrical arrangement and the field scanning is similar to the well-known Hanle effect (zero field level crossing), therefore we call it a Hanle effect configuration. A sketch of the experimental arrangement is given in Fig. 5 .

Of course, also in our experiment the Hanle effect is present: it causes a depolarization of the emitted fluorescence light when the magnetic field $\boldsymbol{B}$ is crossing zero. But when looking for the total (unpolarized) fluorescence intensity, we observe no fluorescence at all for the crossing point, indicating that no excitation of the upper levels take place. From the point of excitation, the difference between $\sigma$ - and $\pi$-polarized light vanishes for zero field, so additional transitions $\Delta m_{F}=0$ can be excited. But the situation is more complicated: additional to optical pumping to the coherent dark state a pumping into incoherent dark states (here all $m_{F}$ states belonging to $F_{\mathrm{g}}=1$ ) takes place. Therefore always competing processes take place, which will be described in more detail in Sec. 3. These processes take place also for $|B| \neq 0$ and are not restricted to the region of the Raman resonance. As an example the excitation of transitions $F_{\mathrm{g}}=2 \rightarrow F_{\mathrm{e}}=1$ with $\sigma$-polarized light is shown together with the level scheme in Fig. 6 . The total fluorescence intensity shows a well pronounced dip around $|B|=0$ due to coherent population trapping.

When two-frequency laser light is used for excitation (we use for this purpose \pm 1 st side bands created by an electro-optical modulator (EOM)), it is possible to create $\Lambda$-systems between $m_{F}$ states belonging to $F_{\mathrm{g}}=2$ and $F_{\mathrm{g}}=1$, and for example, $F_{\mathrm{e}}=2[6]$. We usually select a certain magnetic field strength $|B|$ and are scanning the frequency splitting. At $|B|=0$ one will observe a

a)

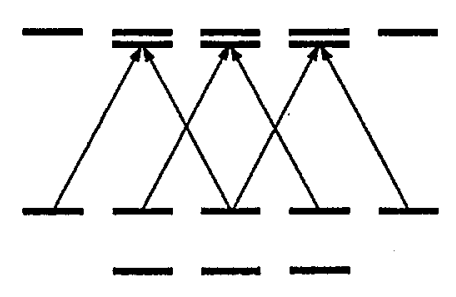

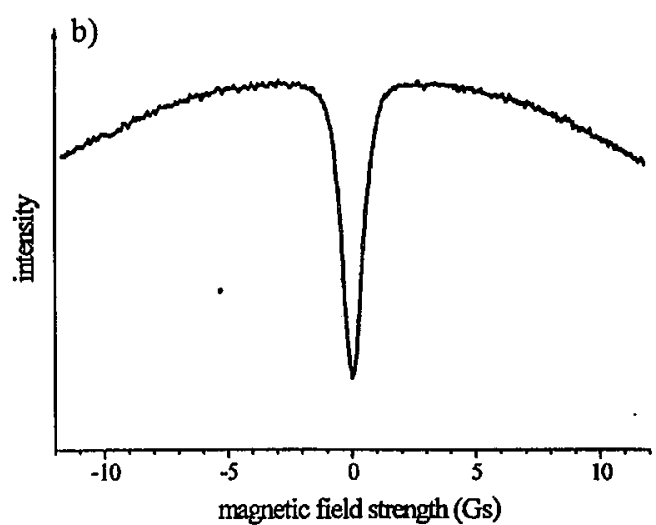

Fig. 6. (a) Level scheme and (b) total fluorescence intensity for $\sigma$-excitation $F_{\mathrm{g}}=2 \rightarrow$ $F_{\mathrm{e}}=1$. 
a)

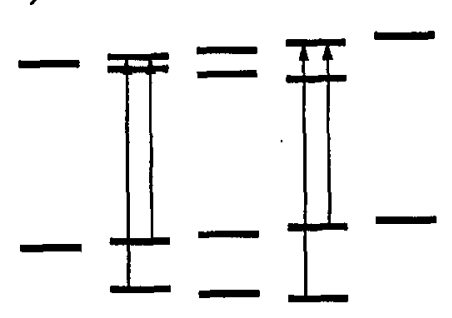

b)

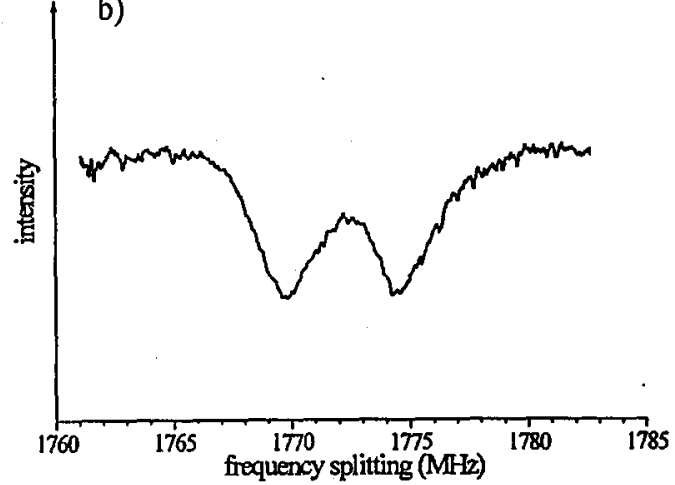

Fig. 7. (a) Level scheme and (b) total fluorescence intensity for $\pi$-excitation $F_{g}=2$, $F_{\mathrm{g}}=1 \rightarrow F_{\mathrm{e}}=1$.

a)

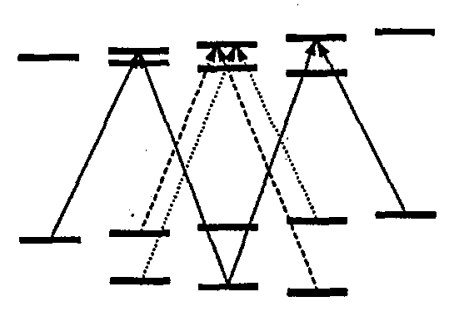

b)

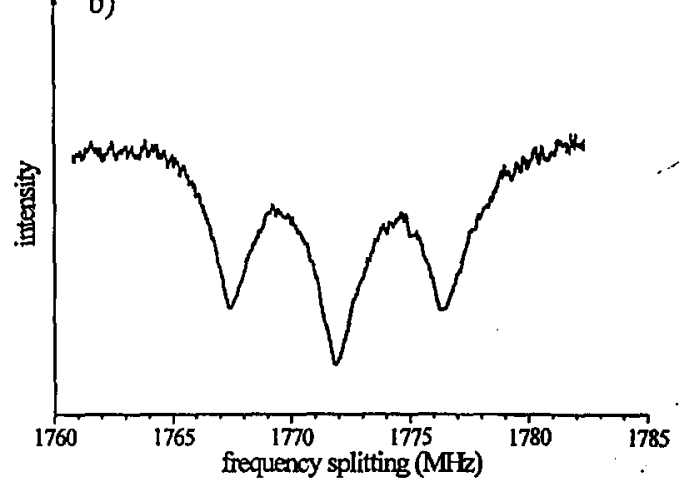

Fig. 8. (a) Level scheme and b) total fluorescence intensity for $\sigma$-excitation $F_{\mathrm{g}}=2$, $F_{\mathrm{g}}=1 \rightarrow F_{\mathrm{e}}=1$. The dashed and the dotted $\Lambda$-systems have the same splitting frequency $\Delta \nu=\left(E_{2}-E_{1}\right) / h=1771.6 \mathrm{MHz}$. Besides the $\Lambda$-systems formed by $\sigma^{+}$- and $\sigma^{-}$-excitation, there exist six other ones excited either by only $\sigma^{+}$- or only $\sigma^{-}$-polarized light. These additional systems have the same frequency splittings as the systems drawn.

single dip around a frequency splitting of $1771.6 \mathrm{MHz}$, and the experiment corresponds to the case described above (the frequency for driving the EOM is then $885.8 \mathrm{MHz}$ ). When choosing $|B|>0$ and $\pi$-polarized excitation, the Raman resonance condition is fulfilled for two different splitting frequencies, forming two different $\Lambda$-systems (Fig. 7), and a double dip is observed. Using $\sigma$-polarized excitation at $|B|>0$, several $\Lambda$-systems having three different frequency splittings are excited and therefore three dips occur (Fig. 8) [7].

\section{A closer look to the establishment of CPT}

As told before, the coherent trap state is usually not the only one dark state within the manifold of $m_{F}$ ground states. Therefore we have to treat another model for establishment of CPT, where we include the loss of atomic population 

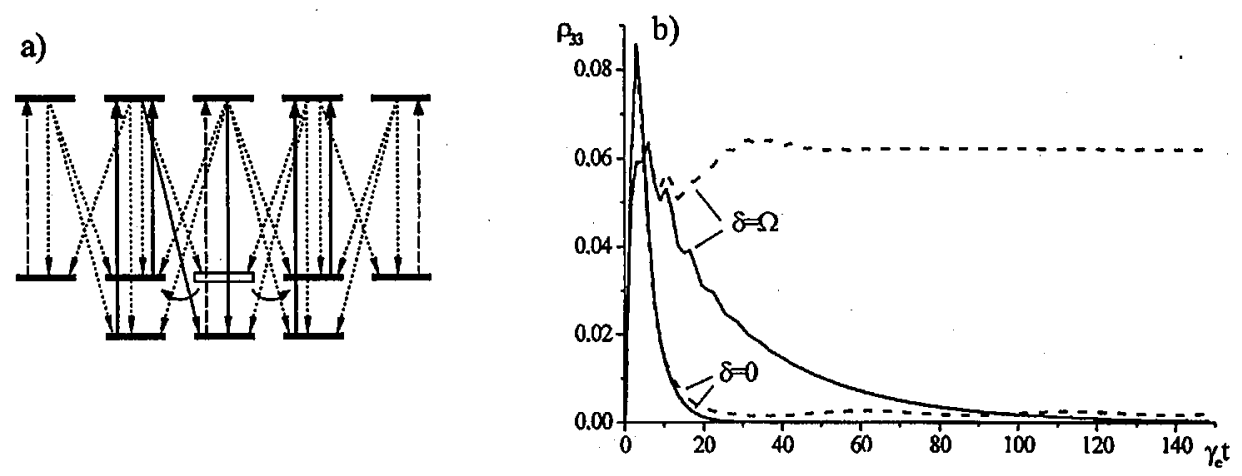

Fig. 9. (a) Level scheme with all excitation and fluorescence channels. Full lines: transitions forming the $\Lambda$-schemes involved, dashed: one-photon transitions excited by laser light, dotted: spontaneous transitions. The level $F_{\mathrm{g}}=2, m_{F}=0$ acts as an incoherent dark state since it is not excited by laser light, but distributes its population to other states by a rate $\gamma_{0, C}$. (b) Calculated time behaviour of $\rho_{33}$ using the simplified level scheme of Fig. 2c. Dashed lines: $\gamma_{0, C}=0.07 \gamma_{3,0}$; solid lines: $\gamma_{0, C}=0.001 \gamma_{3,0}$. The population is shown for two detunings, $\delta_{\mathrm{R}}=\Omega$ and $\delta_{\mathrm{R}}=0$. In case of pumping to the coherent dark state $\left(\delta_{\mathrm{R}}=0\right)$ the time behaviour is marginally influenced by the natural trap state $F_{\mathbf{g}}=2, m_{F}=0$.

by introducing a state $|0\rangle$ to which the atoms can decay from $|3\rangle$. This state may be coupled to one or both ground states by a small rate, allowing in this case the atoms to relax back to excitable states (Fig. 2c). In Fig. 9a the level scheme is shown again in which additional to the excitations all possible fluorescence transitions are indicated. The state $F_{\mathrm{g}}=2, m_{F}=0$ is not excited by one of the light fields and is acting as an incoherent dark state. But the relaxation $\gamma_{0, C}$ takes care for recycling of the population of this state. In Fig. $9 \mathrm{~b}$ the time development of the population of the excited state, $\rho_{33}$, is calculated for two different values of coupling $\gamma_{0, C}$. For a large coupling (e.g. $\gamma_{0, C}=0.07 \gamma_{3,0}$, which corresponds to the influence of the earth magnetic field) and large detuning from the Raman resonance $\left(\delta_{\mathbf{R}}=\Omega\right)$ the population of state $|3\rangle$ becomes nearly constant after short time (dashed curve), but for $\gamma_{0, C}=0.001 \gamma_{3,0}$ all the population is trapped in $F_{\mathrm{g}}=2, m_{F}=0$. CPT is practically not present for this case. The situation changes for $\delta_{\mathrm{R}}=0$ : now additionally to pumping to $F_{\mathrm{g}}=2, m_{F}=0$ pumping to $|N C\rangle$ takes place. In this case the coupling $\gamma_{0, C}$ does not influence much the time behaviour, since it is dominated by coherent processes. Experimentally we have checked these predictions by measuring the fluorescence intensity of an atomic beam along the interaction region with laser light (beam diameter ca. $8 \mathrm{~mm}$ ) according to Fig. 10 .

When the interaction region is not shielded from the earth's magnetic field and the detuning $\delta_{\mathrm{R}}$ is large, one observes - as usually - an intensity which is approximately constant over the whole fluorescence zone (Fig. 11a). This behaviour is due to the strong coupling $\gamma_{0, C}$ by the Larmor precession in the earth magnetic field ( $c a .0 .7 \mathrm{Gs}$ ). In case of CPT $\left(\delta_{\mathrm{R}}=0\right)$ the fluorescence intensity at the beginning of the interaction region, where the atoms are entering the laser beam, is the 


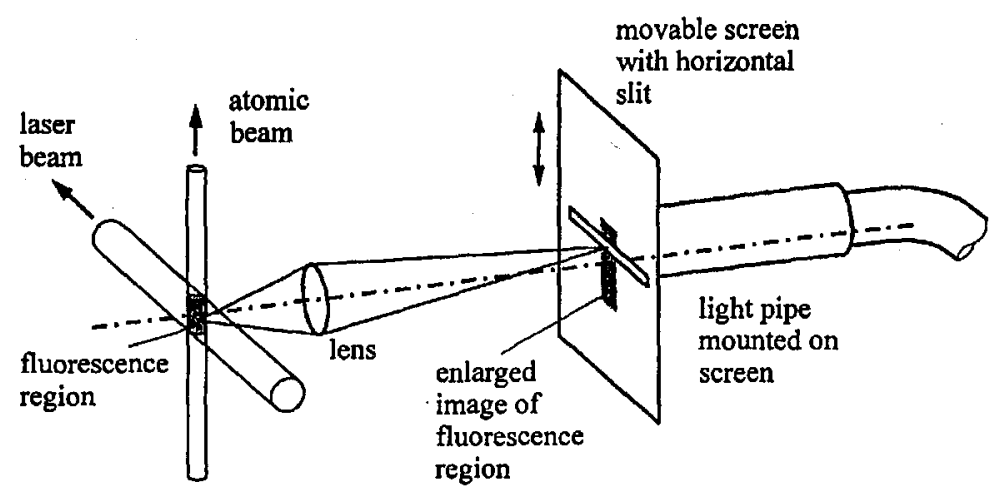

Fig. 10. Experimental arrangement for measuring the time development of the fluorescence intensity.

a)

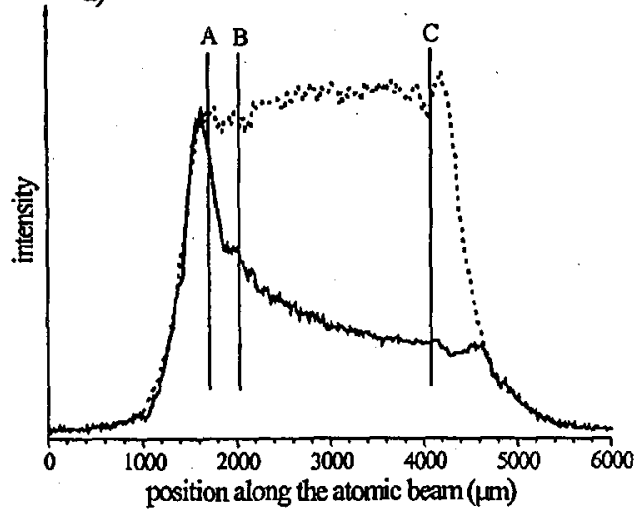

b)

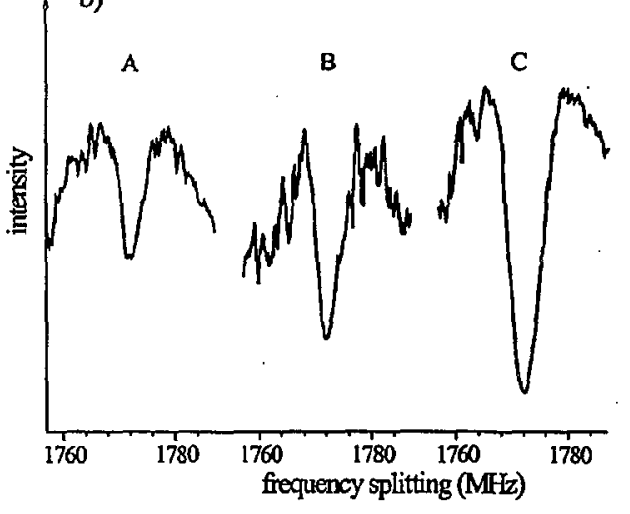

Fig. 11. (a) Fluorescence intensity along the path of the atomic beam. Dotted curve: $\delta_{\mathrm{R}}$ outside the CPT-dip; solid line: $\delta_{\mathrm{R}}=0$. (b) Intensity in dependence of the Raman detuning at different positions $(A, B, C)$ along the fluorescence path. Earth magnetic field present $\left(\gamma_{0, C}\right.$ large $)$.

same as in the case of large detuning. But now after some fluorescence cycles a large number of atoms is pumped into a coherent dark state, and the fluorescence decreases dramatically, but the coupling is still remarkable and does not lead to a complete vanishing of fluorescence. Please note that the position along the atomic beam does not correspond directly to a timescale due to the velocity distribution of the atoms in the beam. Along the fluorescence path in Fig. 11a the intensity has been recorded in dependence on the splitting frequency of the exciting laser fields for three positions $A, B, C$. Indeed, the CPT-dip is most pronounced at the end of the fluorescence zone (Fig. 11b). When shielding the interaction region by some cylinders of $\mu$-metal, the coupling $\gamma_{0, C}$ gets much smaller, and atoms which are pumped to the dark state $F=2, m_{F}=0$ stay there for longer time. The dotted curve in Fig. 12 (the Raman detuning $\delta_{\mathrm{R}}$ outside the CPT-dip) therefore shows a 


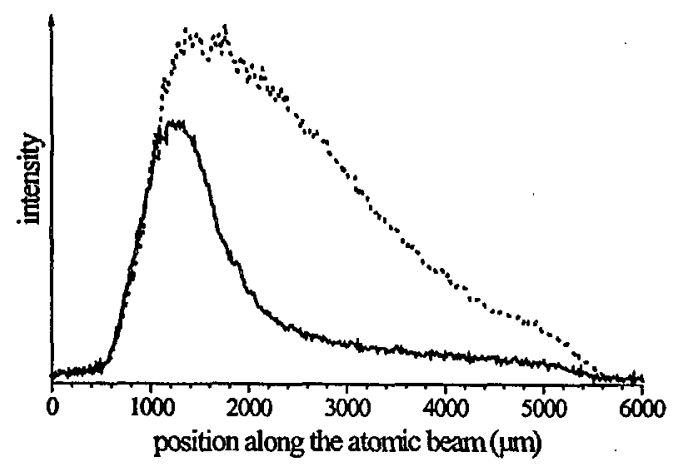

Fig. 12. Fluorescence intensity along the path of the atomic beam. Dotted curve: $\delta_{R}$ outside the CPT-dip; solid line: $\delta_{\mathrm{R}}=0$. The earth magnetic field is shielded ( $\gamma_{0, C}$ small).

decrease in intensity along the fluorescence path. In case of $\delta_{R}=0$ additionally the coherent dark state is present, and the atomic population is trapped in one of the dark states much earlier (full line in Fig. 12) [8].

We can now understand the real case of establishment of a dark resonance within the $m_{F}$ manifold of the sodium $D$-lines as concurring optical pumping into incoherent and coherent dark states, where some relaxations exist. The width of the CPT-dip is mostly determined by relaxation processes and by the intensity of the exciting fields. The smaller the relaxations, the smaller the pumping rate for coherent trapping can be and still have a noticeable influence. Even though there exist no theoretical lower intensity limit for CPT within an ideal $\Lambda$-system, in practice the pumping rate to the CPT state has to be comparable to the pumping rate into the natural dark states [8]. The shapes of the CPT resonances for the Hanle effect configuration have been investigated in Refs. [9-11].

When investigating not the fluorescence intensity but the intensity which is transmitted through an absorption cell, one gets increased transparency in case of establishing CPT. This effect is called electromagnetically induced transparency (EIT) and has been studied also extensively [12-15].

\section{CPT within closed interaction contours}

In Fig. 2 the normally used $\Lambda$-scheme is shown. If an additional electromagnetic field is present, driving transitions between both ground states $|1\rangle$ and $|2\rangle$, all three levels are connected by electromagnetic waves and we can speak about a closed interaction contour. In case of the sodium $D_{1}$-line, this would be a microwave field with $1771.6 \mathrm{MHz}$ (Fig. 13a). One can get a closed interaction contour also in multilevel systems, and in some cases it is possible to create closed systems only using optical transitions. In Fig. 13b,c some possibilities of creating closed systems within the hyperfine states of the sodium $D_{1}$-line are shown.

It was shown first by Buckle et al. [16] that in case of closed interaction contours the phase factors of the wave functions, which usually can be neglected, play an important role. These phase factors are determined by the phases of the involved atomic dipoles and the phases of the coherent electromagnetic fields, and 

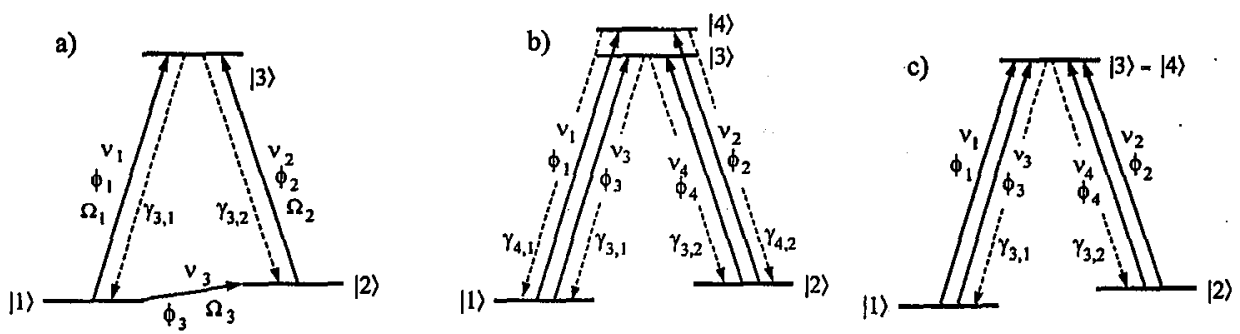

Fig. 13. Closed interaction contours within the manifold of the sodium $D_{1}$-line hyperfine states. (a) System closed by a microwave field, (b) four optical transitions form a four-level double $\Lambda$-system, (c) if for both $\Lambda$-systems the upper level is the same, one gets a degenerated double $\Lambda$-system.

interference effects in the dynamics of the populations appear. In this paper an atom under influence of electromagnetic waves forming a closed interaction contour is called a "one atom interferometer". In this first paper spontaneous emission was neglected. In the next years there was only one experiment performed [17] showing that atoms can be reanimated to absorb photons only by a microwave field of a certain phase compared to the two-frequency laser light pumping the atoms in the coherent dark state. But here the experiment was performed on an atomic beam and the microwave field and the optical fields did not act at the same place.

A more detailed theoretical paper by the group of Matisov [18] was concerned with general multilevel closed contours, including spontaneous relaxations. Also here it was stated that a non-absorbing coherent superposition of two states (in which eventually the population can be trapped) can be established (for the double $\Lambda$-system of Fig. 13b) only for certain values of a total phase $\Phi$ :

$$
\Phi=\left(\theta_{1}-\theta_{2}\right)-\left(\theta_{3}-\theta_{4}\right)
$$

with $\theta_{m}=\chi_{m}+\varphi_{m}(m=1 \ldots 4)$, where $\chi_{m}$ are the phases of the corresponding matrix elements of the atomic dipole moment, $\varphi_{m}$ are the phases of the electromagnetic waves. Since the values of $\chi_{m}$ depend only on the selected transitions, one gets at the interaction region $\Phi=\Phi_{\text {atom }}+\left(\varphi_{1}-\varphi_{2}\right)-\left(\varphi_{3}-\varphi_{4}\right)$. In this way the establishment of CPT can be controlled by changing the phase of one of the optical waves.

For the most simple case of Fig. 13a one can choose the properties to give simple analytical solutions: $\Omega_{1}=\Omega_{2}=\Omega, \gamma_{1}=\gamma_{2}=\gamma$ (a symmetrical $\Lambda$-system), and one gets in case of fulfillment of a three-photon resonance condition $\Delta \nu=$ $\left(\nu_{1}-\nu_{2}\right)-\nu_{3}=0$ (see Ref. [18])

$$
\rho_{33}=\frac{4 \Omega^{2} \Omega_{3}^{2} \sin ^{2} \Phi}{\Omega_{3}^{2} \gamma^{2}+4\left(\Omega^{2}-\Omega_{3}^{2}\right)^{2}+12 \Omega^{2} \Omega_{3}^{2} \sin ^{2} \Phi} .
$$

One can easily see that $\rho_{33}=0$, that means establishment of coherent population trapping, can occur only for certain values $\Phi=n \pi(n=0,1,2, \ldots)$, while the highest population is observed for $\Phi=(2 n+1) \pi / 2(n=0,1,2, \ldots)$, and the coherent trap state is completely destroyed for the latter case. One of the features of Eq. (4) is that for higher intensities the phase dependence is more pronounced, 
that means the phase region where noticeable trapping is taking place, gets smaller. The FWHM of the CPT region gets

$$
\Delta \Phi=\frac{2}{\sqrt{\gamma^{2} /\left(2 \gamma^{2}+12 \Omega^{2}\right)}} .
$$

We have investigated this behaviour first on the scheme of Fig. 13b since it is easier for us to apply optical frequencies [19]. Each pair of frequencies has been produced from a separate carrier frequency, generated by a dye laser system. In order to avoid optical interference, we have sent the two pairs to an atomic beam from opposite directions. The experimental setup is shown schematically in Fig. 14. The total phase is given by

$$
\Phi=\Phi_{\text {atom }}+\left(\varphi_{1}-\varphi_{2}\right)-\left(\varphi_{3}-\varphi_{4}\right)
$$

and a four-photon resonance condition

$$
\Delta \nu=\left(\nu_{1}-\nu_{2}\right)-\left(\nu_{3}-\nu_{4}\right)=0
$$

has to be fulfilled. For observation of the effect, one has to establish for a certain time stable phase relations between the four waves. In the first moment it seems to be impossible to fulfill this condition, since each of the four waves has a frequency of $c a .5 \times 10^{14} \mathrm{~Hz}$ and a line width of $c a .10^{6} \mathrm{~Hz}$. But a closer look to Eq. (7) shows that only the differences of frequencies have to be stable - and we can produce these differences very accurately and phase-stable by means of an electro-optical modulator (EOM). Moreover, when driving both EOM's with the same frequency (e.g. by means of two frequency generators referenced to the same time basis), we get an absolute correlation between the phases of the four waves at the places of the EOM's. During its way to the fluorescence zone, certain but constant phase differences occur due to the optical distance from the EOM's, so the waves at

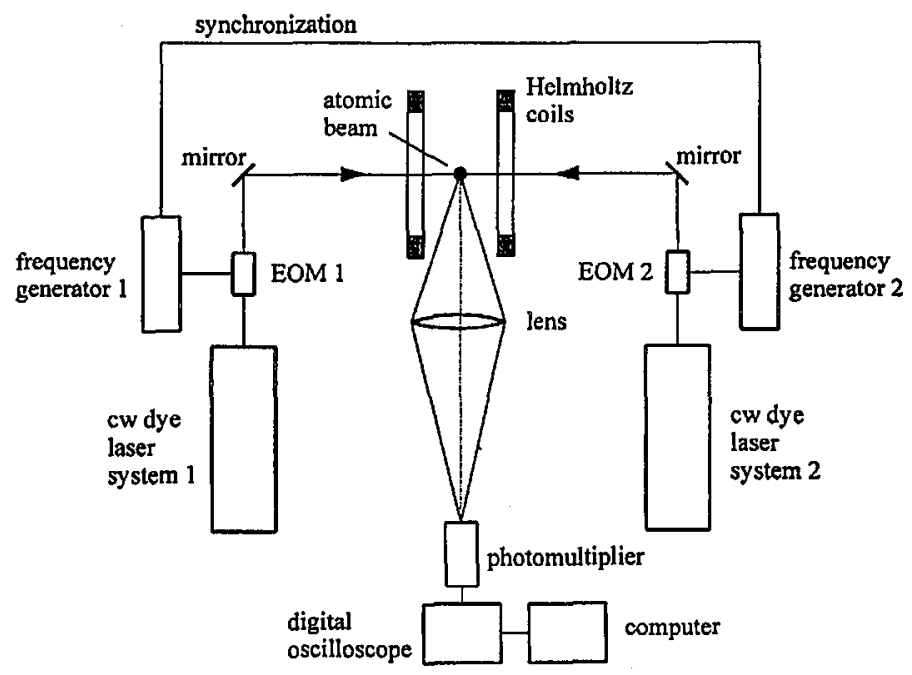

Fig. 14. Experimental investigation of phase-dependent population trapping on a sodium atomic beam (atomic beam direction perpendicular to the drawing plane). 

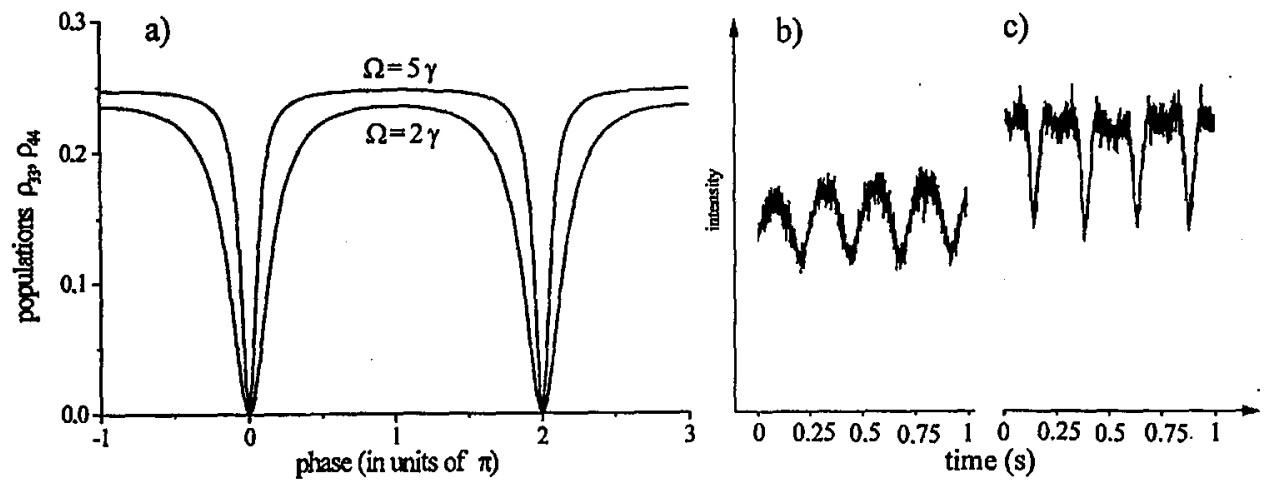

Fig. 15. (a) Calculation of the upper state populations $\rho_{33}, \rho_{44}$ in dependence of the total phase $\Phi$. (b,c) Experimentally observed fluorescence intensity while changing the total phase with time for (b) $I=10 \mathrm{~mW} / \mathrm{cm}^{2}$, (c) $I=100 \mathrm{~mW} / \mathrm{cm}^{2}$. The phase increases with time like $\Delta \Phi=8 \pi t$.

the interaction region have a temporarily constant, but not freely chosen total phase $\Phi$. In order to vary temporarily the phase, we apply to one of the EOM's a slightly different frequency than to the other one, say $886 \mathrm{MHz}$ and $886 \mathrm{MHz}+$ $5 \mathrm{~Hz}$. In such a case the four photon resonance condition is not hurted substantially, but the phase is now varying linearly with time as $\frac{2 \pi}{10} t$.

Figure 15 shows the fluorescence signal observed from the interaction region of the atomic beam and the two pairs of laser frequencies as a function of time and therefore of the time-dependent phase. As can be seen comparing Fig. 15b and c, the phase dependence indeed is more pronounced for higher intensity of the beams. More detailed calculations of the double $\Lambda$-system treated here showed [19] that there is also a dependence on the relative strengths of the transitions involved, expressed by the ratio

$$
r=\frac{\Omega_{1} / \Omega_{2}}{\Omega_{3} / \Omega_{4}}
$$

The coherent dark state shows the most pronounced phase dependence for $r=1$, but this ratio is not realized in case of the scheme of Fig. 13b. Due to the special way of creating our frequency pairs by an EOM, the \pm 1 st side bands have always the same intensity, but the dipole moments of the transitions involved are different, so one cannot choose arbitrary ratios $\Omega_{1} / \Omega_{2}$ or $\Omega_{3} / \Omega_{4}$. But even in our case $r=1$ is observable when investigating a degenerated $\Lambda$-system like the one of Fig. 13c. Figure 16a shows the calculated shape of the CPT-dip for different values of $r$, Figs. $16 \mathrm{~b}$ and $\mathrm{c}$ show experimental curves. In case of the degenerated system the contrast is much higher. But in all experimental curves one can see that the intensity, even for $\Phi=0$, does not go down to the theoretically predicted value of zero. This can be understood by additional excitation of states which are not participating in forming the double $\Lambda$-system, and due to the fact that the interaction region was not shielded against the earth magnetic field, so the lower states of these single photon transitions were filled from the reservoir of non-excitable incoherent dark states. 

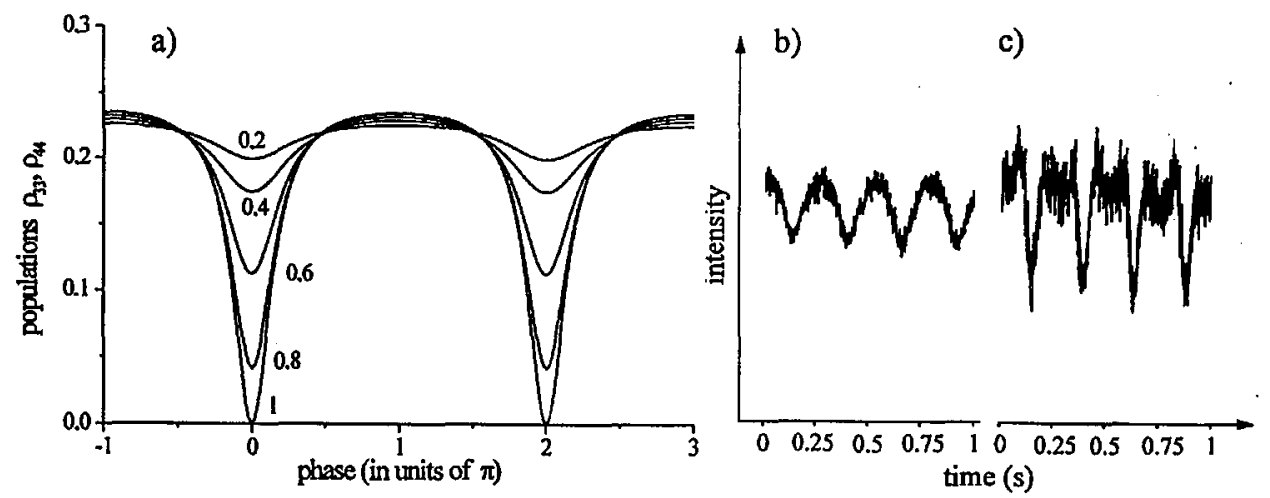

Fig. 16. (a) Calculation of the upper state populations $\rho_{33}, \rho_{44}$ in dependence of the total phase $\Phi$ for different values of the ratio $r$. (b,c) Experimental results for (b) $r=1 / 3$ and (c) $r=1$ (degenerated four-level system). The phase increases with time like $\Delta \Phi=8 \pi t$.
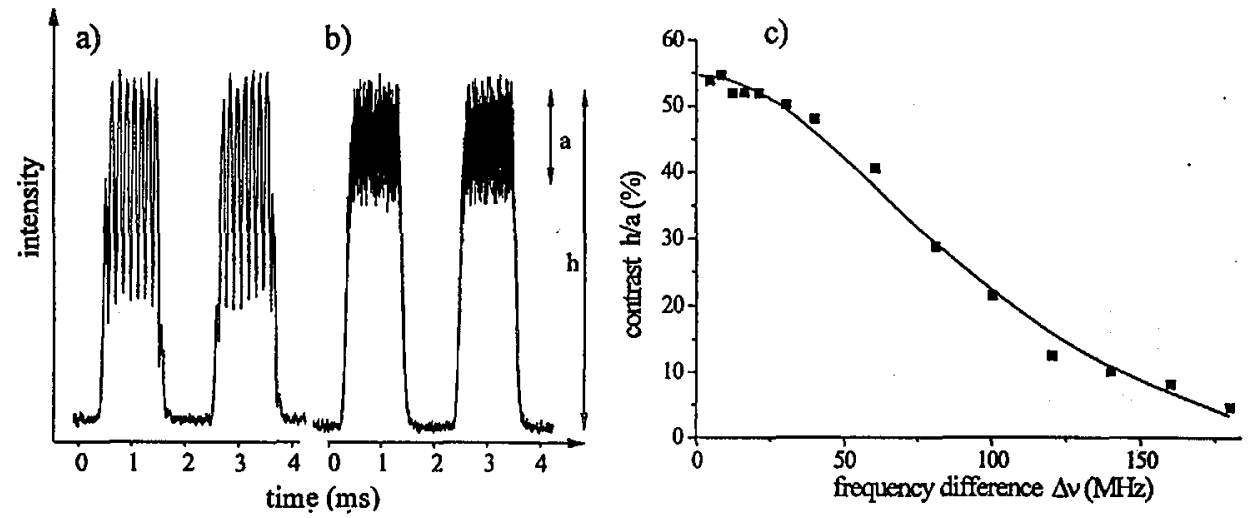

Fig. 17. Phase dependent fluorescence signal for (a) $\Delta \nu=10 \mathrm{kHz}$, (b) $\Delta \nu=100 \mathrm{kHz}$. For an easier determination of the contrast $h / a$, the atomic beam was chopped with a frequency of $\approx 500 \mathrm{~Hz}$. (c) The dependence of the contrast on the frequency difference $\Delta \nu$. Full line: calculated behaviour.

What happens now when the phase is varying much faster in time (again the four-photon resonance should not be harmed substantially), e.g. with frequencies going up to $100 \mathrm{kHz}$ ? In such situations only for very short time the atom feels a phase leading to establishment of CPT - it becomes a transient effect. Figures $17 \mathrm{a}$ and $\mathrm{b}$ show the change in the fluorescence intensity with time for different frequencies of changing the total phase. It can be clearly seen that the contrast gets worse with increasing frequency. For a better determination of the contrast, now also the atomic beam was chopped. The dependence of the contrast on frequency fits well to our theoretical model (Fig. 17c) [20]. 


\section{Phase dependent electromagnetically induced transparency}

The same behaviour as observed when investigating the fluorescence of an atomic beam when shining in two pairs of laser frequencies should be observed in principle when sending the composition of all four frequencies through an absorption cell. In this case the transmitted intensity should depend strongly of the total phase $\Phi_{\text {waves }}$. But for practical reasons the experiment is not as simple as it might look like. First of all, the laser beam after an EOM does contain not only the two first order side bands used for excitation of the $\Lambda$-schemes, but also the carrier frequency ( $c a .1 / 3$ of the total intensity) and second order side bands (even with lower intensity). When exciting an atomic beam - the experiment is nearly Doppler-free in that case - the additional side bands are not in resonance with any atomic transitions and therefore do not play a role. But in a cell these frequencies would be in resonance with Doppler-shifted transitions corresponding to certain velocity groups within the atomic vapour, increasing in this way the fluorescence background. Therefore we had to suppress the non-useful side bands by a Fabry-Perot mode filter which allows only the first order side bands to pass. The second reason is that we had to compensate carefully the earth magnetic field in order to get the most possible contrast, minimizing in this way the Larmor coupling between the magnetic hyperfine states.
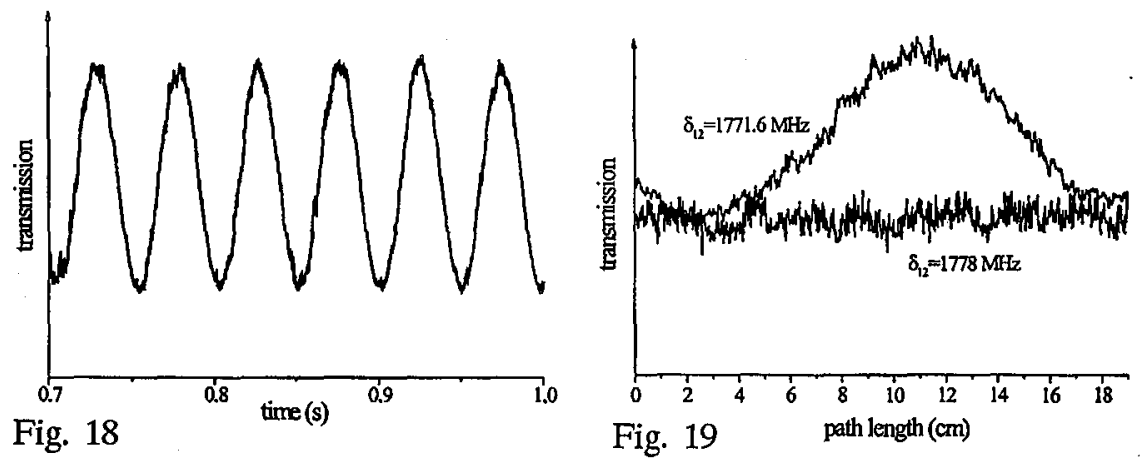

Fig. 18. Phase-dependent electromagnetically induced transparency: variation of the phase with time (increase like $\Delta \Phi=40 \pi t$ ).

Fig. 19. Phase-dependent electromagnetically induced transparency: variation of the phase when increasing the way of one frequency pair to the cell (the phase increases like $\Delta \Phi=(2 \pi / 16.9) x[\mathrm{~cm}])$.

Indeed it was possible to observe a strong dependence of the transmitted intensity on the total phase [21]. The phase could be changed as before by applying slightly different frequencies to the two EOM's or by varying the way of one frequency pair to the cell by shifting a stack of triple mirrors. The results are shown in Figs. 18 and 19. Additionally, in Fig. 19 two curves for different splittings of the pairs of frequency are shown: when the splitting is outside the CPT window, one does not observe a dependence on the phase but gets always absorption. 
The case of phase dependent EIT is of special interest since the electromagnetic waves do not only influence the properties of the absorbing medium, but these influence vice versa also the light waves: the refraction index (and therefore the light velocity within the medium) is dependent on the phase and on the spatial coordinate along the absorption path. On the other hand, in a medium the total phase of the four waves is also varying along the light path.

\section{Conclusion}

The manifestation of CPT has been investigated systematically by our group using as a model system the hyperfine manifold of the sodium $D_{1}$-line. A large number of applications of CPT has been shown by different groups, e.g. for measuring extremely small magnetic fields [22,23], or for laser cooling by velocity selective coherent population trapping processes [24, 25]. Moreover, it seems that "dark resonances" are present in a large number of previous experiments, without calling this effect explicitly.

\section{Acknowledgments}

I would like to thank all co-workers which have been involved in the presented investigations: C. Neureiter, E. Korsunsky, R. Höller, R. Gaggl, W. Maichen, F. Renzoni, N. Leinfellner, W. Scherf, and A. Huss, as well as the guests I. Mazets, J. Xu, and S. Balushev. The cooperations with Prof. E. Arimondo and Prof. B. Matisov are also gratefully acknowledged, as well as the continuous support of the head of our institute, Prof. H. Jäger. I also would like to thank Prof. G. Alzetta for his interest on our work. Special thanks are devoted to Prof. J. Heldt who invited me to give a lecture at the Kolos memory symposium within the frame of the German-Polish joint conference on "Modern Optics", 1998.

The research work was funded by Austrian Science foundation, project numbers S 6508 and P 12894, exchange programs of the Technical University of Graz and the Austrian-Italian scientific-technical agreement.

\section{References}

[1] G. Alzetta, A. Gozzini, L. Moi, G. Orriols, Nuovo Cimento 36, 5 (1976).

[2] G. Alzetta, L. Moi, G. Orriols, Nuovo Cimento 52, 209 (1979).

[3] E. Arimondo, G. Orriols, Lett. Nuovo Cimento 17, 33 (1976).

[4] H.R. Gray, R.W. Whitley, C.R. Stroud Jr., Opt. Lett. 3, 218 (1978).

[5] E. Arimondo, in: Progress in Optics, Ed. E. Wolf, Vol. 35, Elsevier, Amsterdam 1996, p. 257.

[6] R. Höller, F. Renzoni, L. Windholz, J.H. Xu, J. Opt. Soc. Am. B 14, 2221 (1997).

[7] W. Maichen, Ph.D. thesis, Technical University of Graz, 1997.

[8] E.A. Korsunsky, W. Maichen, L. Windholz, Phys. Rev. A 56, 3908 (1997).

[9] F. Renzoni, W. Maichen, L. Windholz, E. Arimondo, Phys. Rev. A 55, 3710 (1997).

[10] F. Renzoni, Ph.D. thesis, Technical University of Graz, 1998. 
[11] F. Renzoni, W. Maichen, J.H. Xu, E. Korsunsky, L. Windholz, Phys. Lett. A 239, 251 (1998).

[12] S.E. Harris, Physics Today (July 1997), p. 36 and references therein.

[13] S. Balushev, N. Leinfellner, E.A. Korsunsky, L. Windholz, Eur. Phys. J. D 2, 5 (1998).

[14] N. Leinfellner, Ph.D. thesis, Technical University of Graz, 1998.

[15] N. Leinfellner, A. Huss, S. Balushev, C. Neureiter, I.E. Mazets, E.A. Korsunsky, L. Windholz, Phys. Scr. 58, 583 (1998).

[16] S.J. Buckle, S.M. Barnett, P.L. Knight, M.A. Lauder, D.T. Pegg, Optica Acta 33, 1129 (1986).

[17] M.S. Shariar, P.R. Hemmer, Phys. Rev. Lett. 65, 1865 (1990).

[18] D.V. Kosachiov, B.G. Matisov, Yu.V. Rozhdestvensky, J. Phys. B 25, 2474 (1992).

[19] W. Maichen, R. Gaggl, E. Korsunsky, L. Windholz, Europhys. Lett. 31, 189 (1995).

[20] W. Maichen, F. Renzoni, I. Mazets, E. Korsunsky, L. Windholz, Phys. Rev. A 53, 3444 (1996).

[21] E.A. Korsunsky, N. Leinfellner, A. Huss, S. Balushev, L. Windholz, Phys. Rev. A, in press.

[22] R. Wynands, A. Nagel, S. Brandt, D. Meschede, A. Weis, Phys. Rev. A 58, 196 (1998).

[23] A. Nagel, L. Graf, A. Naumov, E. Mariotti, V. Biancalana, D. Meschede, R. Wynands, Europhys. Lett., in press.

[24] A. Aspect, E. Arimondo, R. Kaiser, N. Vansteenkiste, C. Cohen-Tannoudji, Phys. Rev. Lett. 61, 826 (1988).

[25] B. Saubamea, T.W. Hijmans, S. Kulin, E. Rasel, E. Peik, M. Leduc, C. Cohen-Tannoudji, Phys. Rev. Lett. 79, 3146 (1997). 